УДК 665.658.62

\author{
ВЕРИФИКАЦИЯ МАТЕМАТИЧЕСКОЙ МОДЕЛИ \\ ГИДРООЧИСТКИ ДИЗЕЛЬНОГО ТОПЛИВА \\ С ПРЕДСТАВЛЕНИЕМ СЕРАОРГАНИЧЕСКИХ ВЕЩЕСТВ \\ В ФОРМЕ ПСЕВДОКОМПОНЕНТОВ
}

\title{
VERIFICATION OF A MATHEMATICAL MODEL OF DIESEL FUEL HYDROTREATING WITH THE REPRESENTATION OF ORGANOSULFUR SUBSTANCES IN THE FORM OF PSEUDOCOMPONENTS
}

\author{
Н.А. Самойлов, В.А. Жилина
}

Уфимский государственный нефтяной технический университет, г. Уфа, Российская Федерация

Naum A. Samoilov, Valeria A. Zhilina

Ufa State Petroleum Technological University, Ufa, Russian Federation e-mail: naum.samoilow@yandex.ru

Аннотация. Из-за отсутствия полномасштабных банков данных по реакционным характеристикам сераорганических примесей сырья гидроочистка дизельных топлив относится к наиболее сложно проектируемым крупнотоннажным процессам нефтепереработки. По мере ужесточения требований к качеству дизельного топлива для увеличения глубины обессеривания сырья существенно увеличилось время контакта очищаемого топлива с катализатором и, как следствие, объем загружаемого в реактор гидроочистки катализатора. Подобная ситуация требует при математическом моделировании процесса гидроочистки достаточно 
корректного учета состава топлива по гидрируемым сераорганическим соединениям.

В статье рассмотрены основные направления по представлению сернистых соединений в исходном сырье процесса гидроочистки: идентификация достаточно представительного набора сераорганических соединений дизельного топлива и разработка банка данных возможных маршрутов реакций, а также объединение компонентов одной группы сераорганических веществ в условный псевдокомпонент, каждый из методов обладает своими специфическими недостатками.

Предложен принцип математического моделирования гидроочистки дизельного топлива путем представления исходного сырья в виде совокупности узких фракций, в каждой из которых содержание разнообразных сераорганических соединений рассматривается как условный псевдокомпонент. В результате анализа нескольких технологических схем реакторных блоков установок гидроочистки при одинаковых закрепленных условиях доказана работоспособность новой модели. Обоснована перспективность двухреакторных систем, характеризующихся раздельным обессериванием потоков. Показано, что при предварительном фракционировании сырья гидроочистки на легкую и тяжелую фракции с выбором оптимальной границы деления фракций можно минимизировать загрузку катализатора в реакторный блок. Выполнен ряд расчетов двухреакторных схем с раздельным гидрированием сырья и исходным сырьем, состоящим из 4, 8 и 16 узких фракций (псевдокомпонентов) с рекомендованными границами деления этих фракций.

Abstract. Due to the lack of full-scale data banks on the reaction characteristics of organosulfur admixtures of raw materials, Hydrotreating diesel fuels is one of the most difficult to design large-capacity oil refining processes. As the requirements for the quality of diesel fuel are tightened to increase the depth of desulfurization of raw materials, the contact time of the purified fuel with 
the catalyst and, as a result, the volume of the catalyst loaded into the Hydrotreating reactor has significantly increased. This situation requires mathematical modeling of the hydrotreatment process to correctly account for the fuel composition of hydrogenated organosulfur compounds.

The article considers the main directions for the representation of sulfur compounds in the feedstock of the Hydrotreating process: identification of a fairly representative set of organosulfur compounds of diesel fuel and development of a data bank of possible reaction routes, as well as combining components of one group of organosulfur substances into a conditional pseudo-component, each of methods has its own specific disadvantages.

The principle of mathematical modeling of diesel fuel hydrotreatment is proposed by presenting the feedstock as a set of narrow fractions, each of which contains a variety of organosulfur compounds considered as a conditional pseudocomponent. As a result of the analysis of several technological schemes of reactor blocks of Hydrotreating plants under the same fixed conditions, the new model is proved to work. The prospects of two-reactor systems characterized by separate desulfurization of flows are proved. It is shown that the preliminary fractionation of Hydrotreating raw materials into light and heavy fractions with the choice of the optimal boundary of the fraction division can minimize the loading of the catalyst into the reactor unit. A number of calculations of two-reactor schemes with separate hydrogenation of raw materials and raw materials consisting of 4,8 and 16 narrow fractions (pseudo-components) with the recommended division boundaries of these fractions were performed.

Ключевые слова: математическое моделирование; дизельное топливо; гидроочистка; сераорганические компоненты; псевдокомпоненты; оптимизация; критерий оптимальности

Key words: mathematical modeling; diesel fuel; hydrotreating; organic sulfur components; pseudo-components; optimization; optimality criterion 
Гидроочистка дизельных топлив относится к наиболее сложно проектируемым крупнотоннажным процессам нефтепереработки из-за отсутствия полномасштабных банков данных, представляющих исходное сырье и необходимых для детального адекватного моделирования процесса физико-химических параметров реакций гидродесульфуризации сераорганических примесей, которые протекают в трехфазной системе: жидкое сырье, водородсодержащий газ, бифункциональные твердые катализаторы, обеспечивающие реализацию гомолитических и гетеролитических реакций $[1,2]$. Степень активности соединений серы в реакциях гидрогенолиза различна и убывает в ряду: меркаптаны $>$ сульфиды $>$ тиофены $>$ бензотиофены > дибензотиофены. При этом наиболее трудно гидрируемые соединения тиофенового ряда сконцентрированы преимущественно в тяжелых фракциях, выкипающих выше $330{ }^{\circ} \mathrm{C}[3]$.

Пока требования к качеству дизельного топлива были низкими, грубая гидроочистка с приемлемой точностью описывалась как некая обобщенная квазигомогенная реакция первого порядка с эффективными кинетическими характеристиками, определенными по изменению содержания общей серы в гидрогенизате во времени $[4,5]$.

По мере ужесточения требований к качеству дизельного топлива и снижения уровня допустимого содержания серы потребовалось существенно увеличить время контакта очищаемого топлива с катализатором и, как следствие, объем загружаемого в реактор гидроочистки катализатора, что привело к переходу от однореакторной схемы процесса к многореакторной, чаще всего двухреакторной схеме с параллельно или последовательно работающими аппаратами [6-14].

Подобная ситуация при математическом моделировании процесса гидроочистки требует перехода к достаточно корректному учету состава топлива по гидрируемым сераорганическим соединениям. Решение этой задачи возможно по нескольким направлениям. 
Первым направлением, наиболее строгим в теоретическом отношении, является попытка идентификации достаточно представительного набора сераорганических соединений дизельного топлива и разработка банка данных возможных маршрутов реакций. Например, в [15] приводится характеристика 38 реакций гидрирования сераорганических компонентов с числом атомов углерода в молекуле от 2 до 12, входящих в характерные для дизельных топлив группы веществ от меркаптанов до дибензотиофенов. Однако если учесть, что в состав дизельной фракции входит большое число сераорганических соединений, то приходится выполнять многочисленные кинетические эксперименты для получения необходимых для моделирования процесса физико-химических характеристик реакций, в первую очередь, констант уравнения Аррениуса (энергии активации и предэкспоненциального множителя). Это направление достаточно сложно реализовать из-за микроконцентраций многих сераорганических веществ в реакционной смеси, что требует использования дорогостоящих препаратов и прецизионного аналитического инструментария.

Второе, менее затратное, направление заключается в объединении компонентов одной группы сераорганических веществ в условный псевдокомпонент, но при этом рассчитываемые константы как уравнения Аррениуса, так и сами константы скорости реакции носят эффективный характер, а формируемые группировки компонентов весьма произвольны. Например, в [16] исследование процесса обессеривания дизельного топлива выполнялось по следующим условным псевдокомпонентам: объединенные сульфиды, объединенные этилбензотиофены, объединенные пропилбензотиофены, объединенные бутилбензотиофены, дибензотиофен, объединенные метилдибензотиофены и объединенные этилдибензотиофены, а в [17] использовалась иная группировка: объединенные алифатические и негетероциклические серасодержащие компоненты, включая тиолы, сульфиды и тиофены, бензотиофен, объединенные бензотиофены, включающие алкильные цепи от 1 до 5 
углеродных атомов, дибензотиофен, объединенные дибензотиофены. Подобные группировки сераорганических веществ обычно рассматривают как псевдокомпоненты, при этом в один псевдокомпонент могут входить различные сераорганические соединения, являющиеся близкими по интенсивности гидрирования. Например, в [17] предложено рассматривать гидроочистку дизельного топлива на основе двух, трех или четырех псевдокомпонентов (рисунок 1).

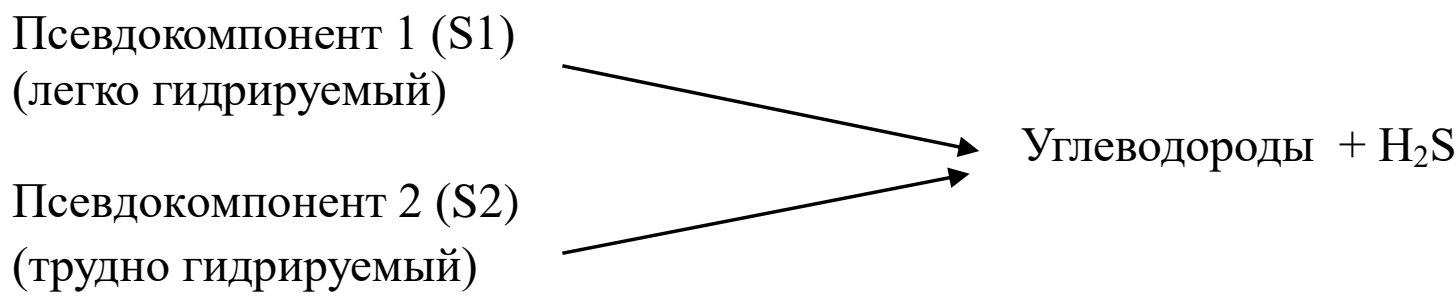

a)

Псевдокомпонент 1 (S1)

(очень легко гидрируемый)

Псевдокомпонент 2 (S2)

Углеводороды + Псевдокомпонент 3 (S3) $+\mathrm{H}_{2} \mathrm{~S}$ (трудно гидрируемый) ( легко гидрируемый)

б)

Псевдокомпонент 1 ( S1) (очень легко гидрируемый)

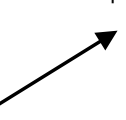

$$
\text { (трудно гидрируемый) }
$$

Псевдокомпонент 2 (S2)
( легко гидрируемый) Углеводороды + Псевдокомпонент 3 (S3) ( трудно гидрируемый)

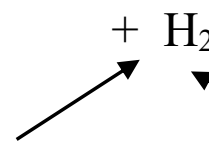
$\mathbf{T}_{\text {(очень трудногидрируемый) }}^{\text {Псевдокомпонент } 4 \text { (S4) }}$

B)

Рисунок 1. Обобщенные схемы химизма процесса гидроочистки с числом псевдокомпонентов два (a), три (б) и четыре (в) [17]

Несмотря на существенные условности, второе направление формирования модели сырья гидроочистки весьма перспективно и может быть использовано для повышения уровня адекватности моделирования как единичных реакторов гидроочистки, так и блоков из двух и более параллельно или последовательно работающих аппаратов, так как во всех 
этих вариантах реакторных блоков общая загрузка катализатора на установку будет одинаковой при прочих равных условиях [18].

Расчеты показали, что в традиционно распространенных вариантах реакторных блоков существенный рост производительности установки и глубины очистки дизельного топлива возможны только при экстенсивных путях решения задачи, а именно за счет значительного увеличения загрузки катализатора в реакторный блок при сохранении активности катализатора и постоянстве остальных технологических параметров [18]. Перспективным интенсивным направлением развития процесса гидроочистки следует считать многореакторные (чаще всего двухреакторные) блоки, где в каждом реакторе осуществляется индивидуальный процесс гидроочистки различных фракций сырья, что позволяет обеспечивать в каждом аппарате оптимальный технологический режим и загрузку наиболее эффективного катализатора. Так, например, при двухреакторной (двухступенчатой) гидроочистке бензиновых фракций каталитического крекинга в реакторе первой ступени осуществляется гидроочистка всего сырья, полученный гидрогенизат фракционируют на легкую (хорошо очищенную фракцию) и тяжелую (плохо очищенную фракцию); тяжелую фракцию подвергают гидроочистке в реакторе второй ступени, а полученный гидрогенизат смешивают с легкой фракцией [19]. В другом примере исходное сырье вначале делят на легкую и тяжелую фракции, которые затем индивидуально подвергают гидроочистке в разных реакторах [20-22]. В обоих случаях авторами получены хорошие результаты при эмпирически подобранных температурных границах деления легкой и тяжелой фракций, например, для дизельного топлива легкая фракция имела пределы выкипания $180-300{ }^{\circ} \mathrm{C}$, тяжелая - 300-360 드, а для бензина граница деления фракций на легкую и тяжелую составляла $70{ }^{\circ} \mathrm{C}$. Наиболее привлекательным можно считать вариант с раздельной гидроочисткой легкой и тяжелой фракций, так как при этом появляется возможность в каждом реакторе применить технологический режим и катализатор, наиболее эффективно адаптируемые к гидроочистке каждой 
фракции. Однако аналитический расчет подобных схем с раздельным гидрированием легких и тяжелых фракций на основе псевдокомпонентов практически невозможен, так как при ректификации исходного сырья соединения одного класса оказываются размытыми по разным фракциям, так, например, нафтенобензотиофены почти в одинаковых концентрациях (0.05-0.07 \%) содержатся в фракциях дизельного топлива $200-250{ }^{\circ} \mathrm{C}, 250$ $300{ }^{\circ} \mathrm{C}$ и $300-350{ }^{\circ} \mathrm{C}$, а бензотиофены наблюдаются во всех фракциях дизельного топлива, в том числе и в легкой фракции $150-200{ }^{\circ} \mathrm{C}[20]$.

Нами предлагается третье направление характеристики сырья гидроочистки по содержанию сераорганических веществ, позволяющее моделировать процесс как при гидроочистке всего сырья, так и отдельных его фракций. Исходное сырье - прямогонная дизельная фракция - с содержанием общей серы 3-0.6 \% масс. в зависимости от природы нефти подвергается разгонке ИТК с выделением узких 5-10 \%-ых фракций, в каждой из которых все сераорганические примеси рассматриваются совокупно без идентификации конкретных соединений и даже классов соединений как один обобщенный сераорганический компонент (псевдокомпонент), характеризуемый анализом на общую серу [23]. Интенсивность гидроочистки каждой узкой фракции может быть оценена на лабораторной или пилотной установке с определением константы скорости реакции гидродесульфуризации соответствующего псевдокомпонента, хотя это достаточно трудоемкая процедура при большом числе узких фракций.

Сократить экспериментальную подготовительную стадию определения физико-химических характеристик для математического моделирования процесса гидроочистки можно, если использовать постепенно формируемый информационный банк данных. К сожалению, длительные пилотные и промышленные исследования процесса гидроочистки сопровождаются многочисленными возмущениями (колебания расходов сырья и его состава по сераорганике, температуры процесса, 
продолжительности контакта сырья с катализатором, дезактивация катализатора), что обычно не позволяет достаточно точно рассчитывать константы скоростей реакций. Например, в [24] в ходе детального анализа работы промышленной трехреакторной установки гидроочистки наблюдалось изменение состава сырья в пределах 1.05-1.25 \% масс., объемной скорости подачи сырья в пределах 1.75-1.80 ч данные по глубине обессеривания сырья по реакторам приводятся без указания времени пребывания сырья в реакторах.

Однако для формирования расчетных закономерностей в ходе симуляции вполне достаточно использовать в некоторой мере произвольные значения этих констант, если их соотношения будут достаточно близкими к реальным.

В работе [24] был выполнен обширный цикл исследований по гидроочистке дизельного топлива на трехреакторном блоке, где в первых двух ректорах осуществлялась предварительная очистка двумя параллельными потоками, а затем совместный гидрогенизат доочищался в третьем реакторе (рисунок 2).
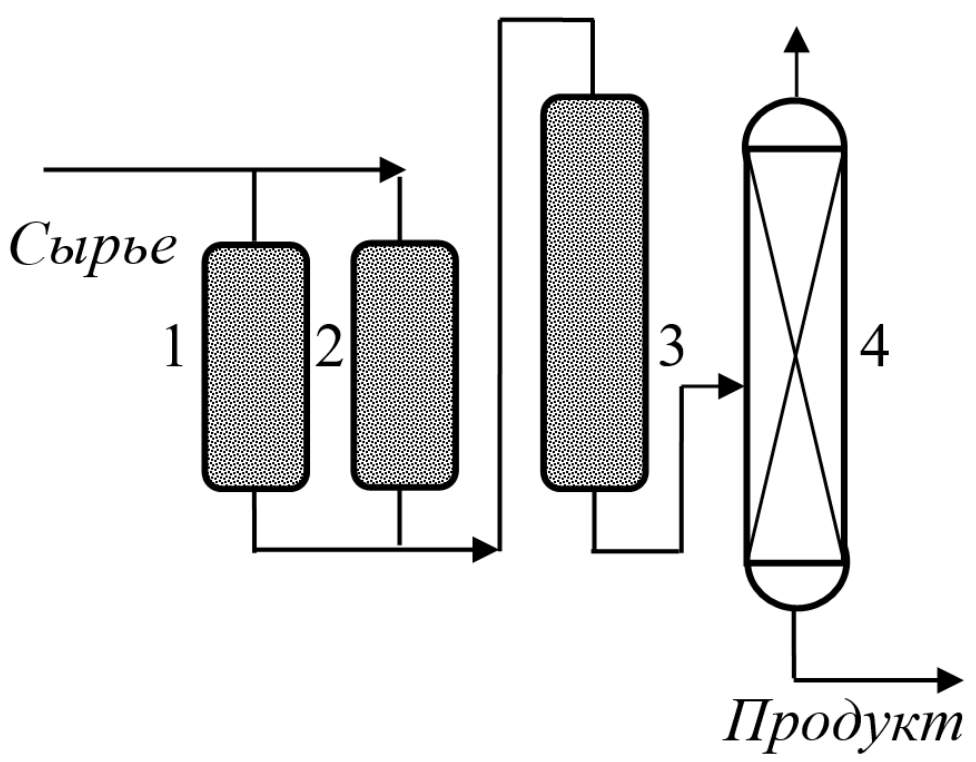

1, 2, 3 - реакторы; 4 - стабилизатор

Рисунок 2. Компоновка реакторного блока

с параллельно-последовательной подачей потоков в реакторы гидроочистки [24] 
При описании экспериментов авторами [24] не указывалось время контакта реакционной смеси с катализатором, поэтому при расчете констант скоростей реакций гидродесульфуризации по данным [20, 24] была принята продолжительность реакции 1 ч, в связи с чем расчетные значения констант носят эффективный характер, но их соотношения могут быть использованы при моделировании процесса.

На рисунке 3 приведены данные по эффективным константам скорости гидроочистки $\mathrm{k}_{1}, \mathrm{k}_{2}, \mathrm{k}_{3}$ по общей сере в реакторах P-1, Р-2 и Р-3. Их соотношения $\mathrm{k}_{1} / \mathrm{k}_{3}$ и $\mathrm{k}_{2} / \mathrm{k}_{3}$, характеризующие относительную интенсивность гидроочистки в различных реакторах, достаточно объективны и лежат в пределах 4-15.

Эффективные константы скорости $\mathrm{k}_{1}$ и $\mathrm{k}_{2}$ в реакторах гидрирования легкой фракции P-1 и Р-2 близки между собой (расхождения в значениях связаны с небольшими отличиями в режиме работы Р-1 и Р-2) и вдвое больше $\mathrm{k}_{3}$ в реакторе гидрирования тяжелой фракции Р-3.

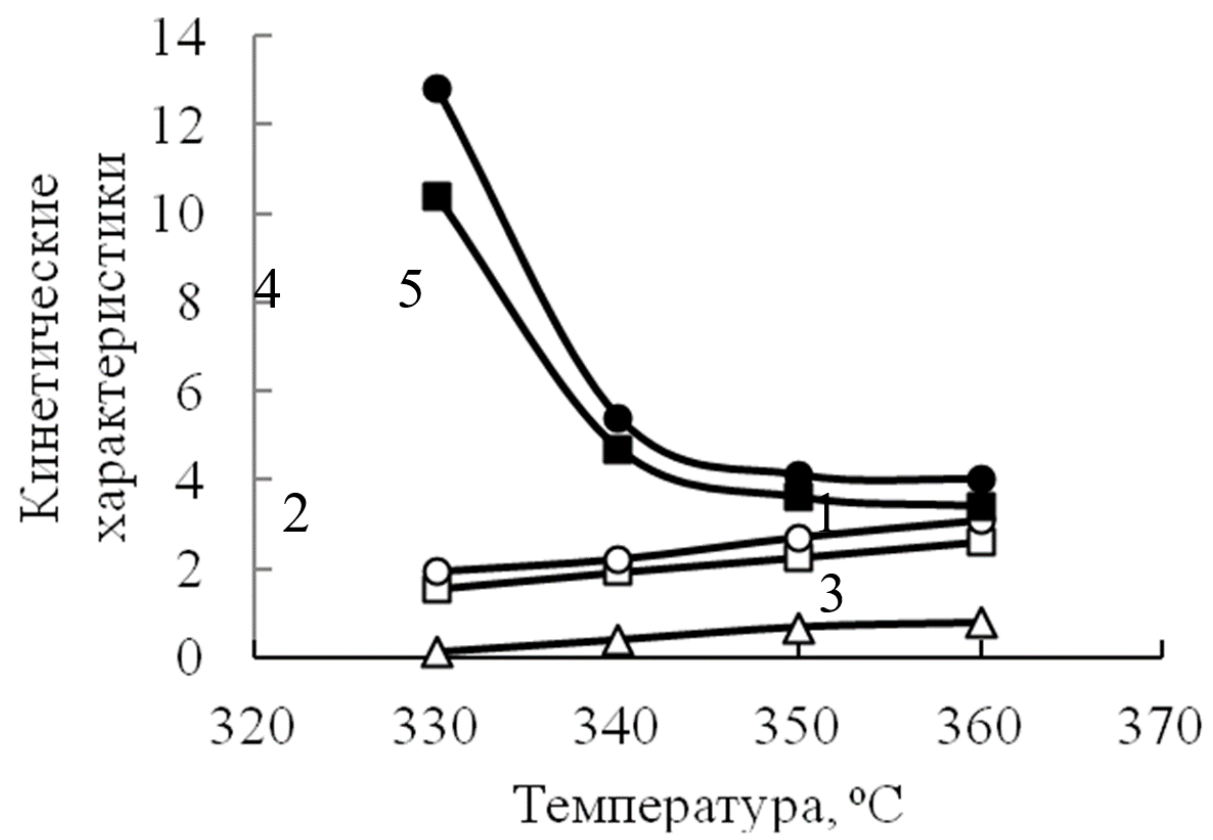

Рисунок 3. Зависимость эффективных констант скорости реакции $\mathrm{k}$ для P-1(1), P-2(2), P-3(3), $\mathrm{k}_{1} / \mathrm{k}_{3}$ (4) и $\mathrm{k}_{2} / \mathrm{k}_{3}$ (5) от температуры [24] 
В таблице 1 приведены аналогичные данные лабораторных экспериментов по гидродесульфуризации дизельной фракции $250-300{ }^{\circ} \mathrm{C}$ по ряду характерных групп сераорганических компонентов, содержащихся в этой фракции, в частности, сульфидов, тиацикланов, тиофенов, бензотиофенов, в количестве $9.456 \%$ масс. (1.7\% масс. в пересчете на массовую долю серы), с использованием метода молекулярно-массового распределения по масс-спектрам отрицательных ионов [20].

Таблица 1. Эффективные константы скорости реакций гидроочистки для групп примесей сераорганики в сырье и гидрогенизате по [20]

\begin{tabular}{|l|c|c|c|}
\hline Соединение & $\begin{array}{c}\text { Степень превращения, } \\
\text { \%, по [20] }\end{array}$ & $\mathrm{k}$ & $\mathrm{k} / \mathrm{k}_{\min }$ \\
\hline Диалкилсульфиды & $\mathbf{9 5 . 0}$ & $\mathbf{2 . 9 9}$ & $\mathbf{1 4 . 9 5}$ \\
\hline Тиамоноцикланы & 93.4 & 2.71 & 13.55 \\
\hline Тиабицикланы & 91.2 & 2.15 & 10.75 \\
\hline Тиатрицикланы & 88.9 & 2.19 & 10.95 \\
\hline Тиатетрацикланы & 86.7 & 2.00 & 10.0 \\
\hline Нафтенотиофены & 70.0 & 1.20 & 6.0 \\
\hline Алкилтиофены & $\mathbf{6 9 . 2}$ & $\mathbf{0 . 2 0}$ & $\mathbf{1 . 0}$ \\
\hline Нафтенобензотиофены & 66.7 & 1.10 & 5.50 \\
\hline Бензотиофены & 66.4 & 1.09 & 5.45 \\
\hline Дибензотиофены & 54.3 & 0.70 & 3.50 \\
\hline $\begin{array}{l}\text { Суммарная } \\
\text { сераорганика }\end{array}$ & 79.6 & 1.59 & 7.95 \\
\hline
\end{tabular}

Результаты выполненного анализа позволяют полагать, что эффективные константы скоростей реакций гидрообессеривания как в промышленных (рисунок 3), так и в лабораторных (таблица 1) условиях соотносятся между наиболее легкогидрируемыми и наиболее трудногидрируемыми компонентами в пределах примерно $15: 1$.

Расчет эффективной константы скорости реакции по изменению общего содержания сераорганики во фракции до и после гидроочистки (соответственно 9.456 и 1.93 \% масс.) дает усредненное значение $\mathrm{k}=1.59$, 
характеризующее псевдокомпонент, эквивалентный сераорганике узкой фракции $250-350{ }^{\circ} \mathrm{C}$.

Полученное соотношение эффективных констант скоростей реакций гидрирования сераорганических веществ различной природы позволило перейти к оценке валидации математической модели гидроочистки с использованием модели сырья на базе понятия псевдокомпонентов.

В ходе математического моделирования реакторного блока с новым принципом характеристики сырья решались следующие задачи:

- оценка близости результатов моделирования к характеристикам аналогичного промышленного блока;

- анализ работы установки гидроочистки;

- критическое сопоставление различных схем компоновки реакционных блоков.

Решение задач обеспечивалось сопоставлением различных технологических схем работы реакторного блока при одинаковых закрепленных условиях: совпадении производительности по очищаемому прямогонному дизельному топливу при одинаковой концентрации общей серы, температуре и давлении в реакторах, расходе водородсодержащего газа и объемной скорости подачи сырья. Исходное сырье делилось на N узких фракций, в каждой из которых находился один сераорганический псевдокомпонент. В ходе моделирования исходное сырье делилось на $\mathrm{N}=16$ узких фракций і по 6.25 \% об., каждая по порядку роста температур их кипения с константами скоростей i-й реакции соответственно равных (17-i).

В качестве допущения был принят режим идеального вытеснения и квазигомогенный характер протекания реакций гидродесульфуризации псевдокомпонентов первого порядка, что позволило корректно упростить расчеты и использовать математическую модель реактора гидроочистки в следующей форме: 


$$
\begin{gathered}
\frac{d C_{S 1}}{d \tau}=-K_{2} C_{S 1} \\
\frac{d C_{S 2}}{d \tau}=-K_{2} C_{S 2} \\
\vdots \\
\frac{d C_{S i}}{d \tau}=-K_{i} d C_{S i} \\
\vdots \\
\frac{d C_{S N}}{d \tau}=-K_{N} d C_{S N}
\end{gathered}
$$

где $C_{\mathrm{Si}}$ и $K_{\mathrm{i}}$ - концентрация i-го сераорганического псевдокомпонента и константа скорости і-й реакции, соответственно.

Модель позволяет рассчитать время контакта реакционной смеси с катализатором $\tau$ для достижения концентрации серы $C_{R S_{\tau}}$ меньше допустимой концентрации серы в очищенном дизельном топливе:

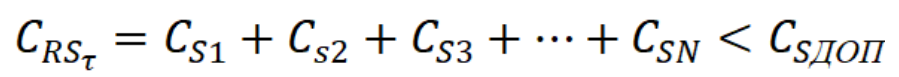

и, соответственно, необходимый объем катализатора, загружаемого в реактор.

В качестве контрольного объекта для оценки валидации разработанной математической модели была принята установка гидроочистки дизельного топлива Л-24-6 Рязанского НП3, параметры работы которой детально изложены в [24]. Реакторный блок этой установки из трех реакторов функционировал с параллельно-последовательной подачей потоков в реакторы гидроочистки (рисунок 2). Поскольку эта схема эквивалентна единичному реактору с загрузкой катализатора, равной суммарной загрузке всех трех аппаратов, то по математической модели был рассчитан единичный реактор, для которого был подобран вариант распределения общей серы $\mathrm{S}_{\mathrm{i}}$ по i-м псевдокомпонентам в ppm (рисунок 4) в форме, учитывающей повышенное содержание трудногидрируемых 
сераорганических компонентов в высококипящих фракциях очищаемого сырья:

$$
\mathrm{S}_{\mathrm{i}}=15000-300 * \mathrm{G}_{\mathrm{i}}+3 * \mathrm{G}_{\mathrm{i}}{ }^{2},
$$

где $\mathrm{G}_{\mathrm{i}}$ - доля отобранных узких фракций от первой по і-ую, \% об.

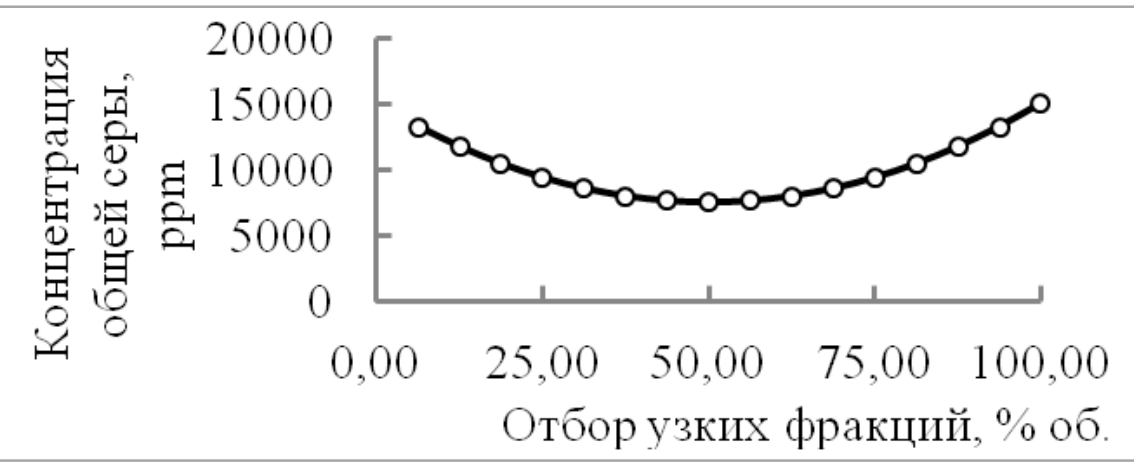

Рисунок 4. Распределение серы по псевдокомпонентам узких фракций

В расчете также была принята насыпная плотность катализатора гидроочистки 750 кг/м³. Результаты моделирования единичного реактора приведены в таблице 2.

Таблица 2. Сравнение расчетных и контрольных данных при моделировании реактора гидроочистки с учетом 16 псевдокомпонентов

\begin{tabular}{|l|c|c|c|}
\hline \multirow{2}{*}{\multicolumn{1}{|c|}{ Параметры }} & \multicolumn{2}{|c|}{ Промышленный блок [24] } & Расчетный блок \\
\cline { 2 - 4 } & Р-1 и Р-2 & Р-3 & Один реактор \\
\hline Расход сырья, $\mathrm{m}^{3} /$ ч & 68 и 68 & 136 & 136 \\
\hline $\begin{array}{l}\text { Концентрация серы на } \\
\text { входе в реактор, ppm }\end{array}$ & $10500-12500$ & 655 & 10000 \\
\hline $\begin{array}{l}\text { Объемная скорость } \\
\text { подачи сырья, ч }\end{array}$ & $1.75-1.80$ & $1.75-1.80$ & 2 \\
\hline Температура, ${ }^{\circ} \mathrm{C}$ & 360 & 360 & 360 \\
\hline $\begin{array}{l}\text { Концентрация серы на } \\
\text { выходе из реактора, } \mathrm{ppm}\end{array}$ & 500 и 810 & 300 & 300 \\
\hline Время реакции, ч & - & - & 1.1 \\
\hline Объем катализатора, $\mathrm{m}^{3}$ & - & - & 74.8 \\
\hline Масса катализатора, $\mathrm{T}$ & 11.9 и 12.6 & 31.4 & 56.1 \\
\hline
\end{tabular}


Загрузка катализатора в единичный реактор, эквивалентный по эффективности промышленным реакторам по [24] (P-1 и Р-2 работают параллельно и суммарный гидрогенизат поступает в Р-3), составила 56.1 т, что близко к суммарной загрузке в три реактора 55.9 т.

Вполне приемлемая сходимость результатов моделирования и данных промышленного реактора (погрешность расчета загрузки катализатора в систему составила около 200 кг) позволила далее перейти к моделированию работы различных схем компоновки реакторных блоков. Было оценено необходимое изменение загрузки катализатора в реакторный блок модернизируемой установки Л-24-6 для обеспечения углубления очистки дизельного топлив от серы с 300 до 10 ppm, а также рассмотрен вариант реконструкции установки, когда исходное сырье предварительно фракционируется на легкую и тяжелую фракции, которые далее гидрируются раздельно (рисунок 5).

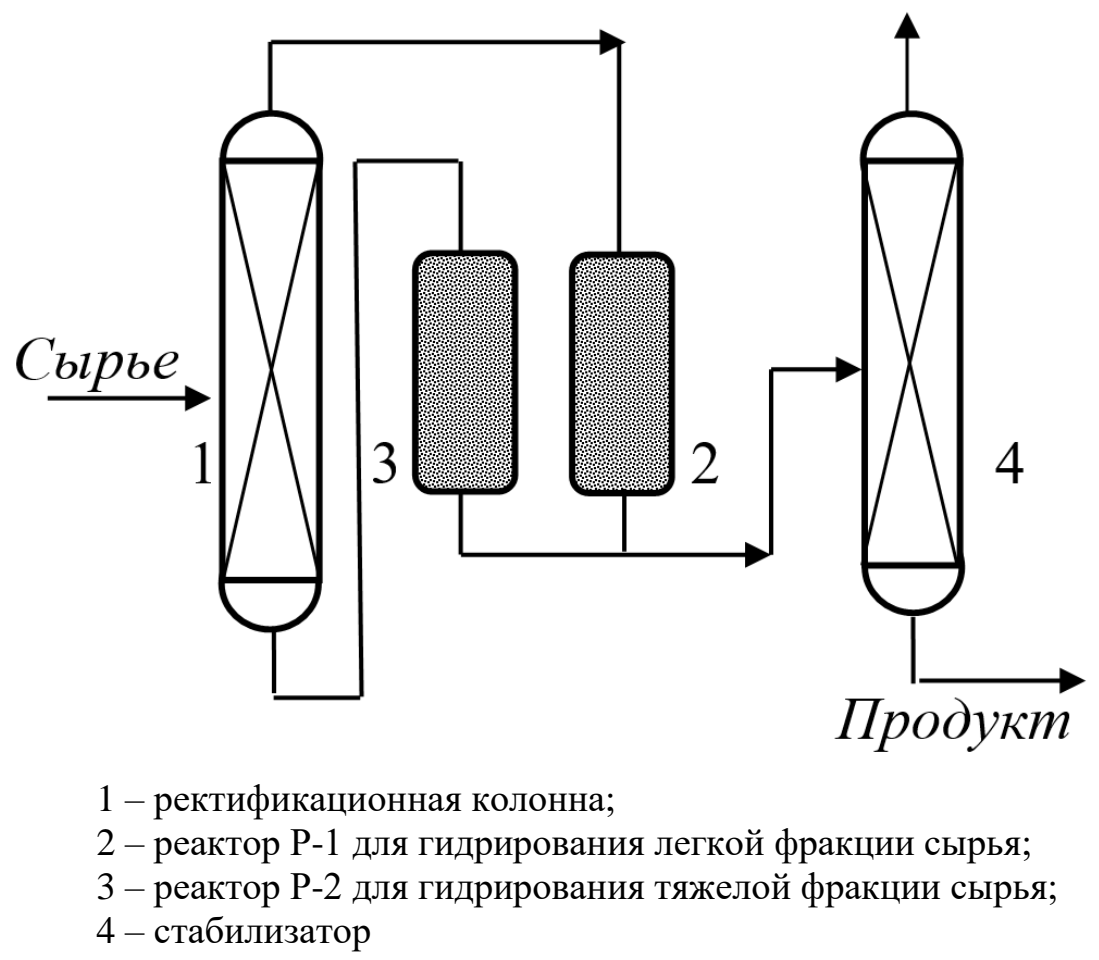

Рисунок 5. Компоновка реакторного блока с раздельным гидрированием сырья, предварительно разделенного на легкую и тяжелую фракции 
Специфика алгоритма моделирования реакторного блока с раздельным гидрированием легкой и тяжелой фракций сырья позволяет просчитывать все возможные варианты первичного фракционирования исходного сырья с загрузкой двух сырьевых фракций в Р-1 и Р-2 как в различном количестве, так и с различным содержанием общей серы, учитывая число узких фракций, попадающих с сырьем в конкретный реактор (таблица 3), при этом каждый вариант обеспечивает достижение необходимого качества очистки дизельного топлива.

Таблица 3. Варианты распределения 16 узких фракций по реакторам: Р-1 (легкая фракция сырья) и Р-2 (тяжелая фракция сырья)

\begin{tabular}{|c|c|c|c|c|c|c|c|c|}
\hline \multirow{2}{*}{ 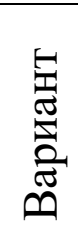 } & \multicolumn{2}{|c|}{$\begin{array}{c}\text { Ввод узких } \\
\text { фракций в } \\
\text { реакторы }\end{array}$} & \multirow{2}{*}{ 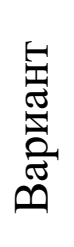 } & \multicolumn{2}{|c|}{$\begin{array}{c}\text { Ввод узких } \\
\text { фракций в } \\
\text { реакторы }\end{array}$} & \multirow{2}{*}{ 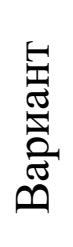 } & \multicolumn{2}{|c|}{$\begin{array}{c}\text { Ввод узких } \\
\text { фракций в } \\
\text { реакторы }\end{array}$} \\
\hline & P-1 & P-2 & & P-1 & $\mathrm{P}-2$ & & P-1 & P-2 \\
\hline 1 & 1 & $2 \ldots 16$ & 6 & $1 \ldots 6$ & $7 \ldots 16$ & 11 & $1 \ldots 11$ & $12 \ldots 16$ \\
\hline 2 & 1,2 & $3 \ldots 16$ & 7 & $1 \ldots 7$ & $8 \ldots 16$ & 12 & $1 \ldots 12$ & $13 \ldots 16$ \\
\hline 3 & $1 \ldots 3$ & $4 \ldots 16$ & 8 & $1 \ldots 8$ & $9 \ldots 16$ & 13 & $1 \ldots 13$ & $14 \ldots 16$ \\
\hline 4 & $1 \ldots 4$ & $5 \ldots 16$ & 9 & $1 \ldots 9$ & $10 \ldots 16$ & 14 & $1 \ldots 14$ & 15,16 \\
\hline 5 & $1 \ldots 5$ & $6 \ldots 16$ & 10 & $1 \ldots 10$ & $11 \ldots 16$ & 15 & $1 \ldots 15$ & 16 \\
\hline
\end{tabular}

Поскольку реализация каждого варианта работы реакторного блока обеспечивается соответствующей индивидуальной загрузкой катализатора, то легко выбрать вариант схемы, решающий поставленную задачу при минимизации загрузки катализатора, рассматриваемой как критерий оптимальности решаемой задачи.

В таблице 4 представлены итоги расчета загрузки катализатора в реакторы по схемам, приведенным на рисунках 2 и 5, при углублении очистки топлива по сере до 10 ppm, что требует четырехкратного увеличения загрузки по схеме параллельно-последовательного гидрирования сырья (рисунок 2) и двухкратного по схеме раздельного гидрирования предварительно фракционируемого сырья (рисунок 5). Необходимо также отметить, что величины загрузки катализатора в 
реакторы P-1 и Р-2 близки, и реакционный блок можно оборудовать одинаковыми аппаратами. При соответствующем изменении объемной скорости подачи сырья и температуры количество катализатора в обоих реакторах несложно уравнять.

Таблица 4. Результаты моделирования реакторных блоков, обеспечивающих снижение содержания общей серы в дизельном топливе до уровня $10 \mathrm{ppm}$

\begin{tabular}{|l|c|c|c|c|}
\hline \multicolumn{1}{|c|}{ Параметры } & $\begin{array}{c}\text { Промышленный } \\
\text { блок по [24] }\end{array}$ & $\begin{array}{c}\text { Модернизация } \\
\text { блока } \\
\text { (рисунок 2) }\end{array}$ & \multicolumn{2}{|c|}{$\begin{array}{c}\text { Реконструкция } \\
\text { блока } \\
\text { (рисунок 5) }\end{array}$} \\
\hline Число реакторов & 3 & 3 & Р-1 & Р-2 \\
\hline Расход сырья, ${ }^{3} /$ ч & 136 & 136 & 109.5 & 26.5 \\
\hline $\begin{array}{l}\text { Концентрация } \\
\text { серы на входе в } \\
\text { реактор, ррт }\end{array}$ & $10500-12500$ & 10000 & 9257 & 13320 \\
\hline $\begin{array}{l}\text { Объемная скорость } \\
\text { подачи сырья, ч }\end{array}$ & $1.75-1.80$ & 2 & 2 & 2 \\
\hline Температура, ${ }^{\circ} \mathrm{C}$ & 360 & 360 & 360 & 360 \\
\hline $\begin{array}{l}\text { Концентрация } \\
\text { серы на выходе из } \\
\text { блока, ррт }\end{array}$ & 300 & 10 & 10 & 10 \\
\hline Время реакции, ч & - & 4.5 & 1.1 & 6.2 \\
\hline $\begin{array}{l}\text { Загрузка } \\
\text { катализатора, т }\end{array}$ & 55.9 & 227.5 & 44.7 & 58.3 \\
\hline
\end{tabular}

На рисунке 6 показано, как изменяется суммарная загрузка катализатора в реакторы установки гидроочистки для реконструируемого блока (таблица 4) в зависимости от границы деления исходного сырья на легкую и тяжелую фракции, каждая из которых характеризуется соответствующим числом узких фракций (все сырье рассматривалось как совокупность 16 узких фракций псевдокомпонентов).

Как следует из рисунка 6, при фракционировании сырья гидроочистки на легкую и тяжелую фракции необходимо обеспечить отбор легкой фракции как совокупности 13 узких фракций, то есть 81.25 \% об. от 
прямогонной дизельной фракции, и гидроочистка обеспечивается 103 т катализатора.

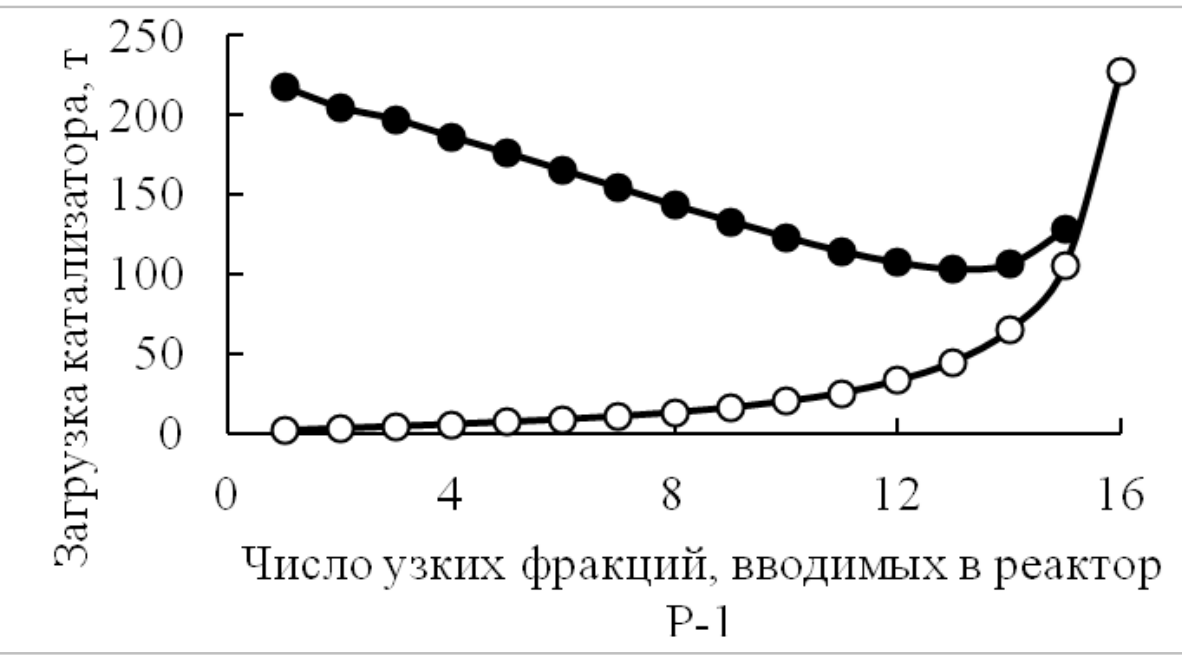

Рисунок 6. Загрузка катализатора в реактор Р-1 (1) и в оба реактора (2) в зависимости от числа узких фракций, вводимых в реактор Р-1

При очистке всего сырья в одном реакторе (или при любой многореакторной схеме, эквивалентной единичному реактору) потребуется загрузить 227 т катализатора. Следует отметить, что расчетный анализ большого числа реакторных блоков с раздельной очисткой предварительно фракционированного сырья, существенно отличающегося по концентрации сераорганических веществ (от 10000 до 30000 ppm), и различными функциями распределения их по узким фракциям показал, что во всех случаях оптимизации задачи отбор легкой фракции при подготовке сырья гидроочистки должен лежать в пределах 75-82 \% об., существенно больших по сравнению с данными [21]. Эта обнаруженная закономерность позволяет существенно упростить экспериментально-аналитическую стадию исследования, предваряющую собственно математическое моделирование, если сократить число узких фракций и соответственно псевдокомпонентов при решении задачи.

Выполнен ряд расчетов двухреакторных схем с раздельным гидрированием сырья при сокращении числа узких фракций и псевдокомпонентов, представляющих исходное сырье, до восьми и 
четырех. При этом в первом случае каждая узкая фракция представляла собой совокупность двух узких фракций 16-кратного деления сырья на узкие фракции, во втором случае - совокупность четырех узких фракций.

Поскольку априори трудно сказать, какие из компонентов, объединяемых в новые расширенные узкие фракции, будут лимитировать процесс их гидрообессеривания, то для гарантии обеспечения глубины очистки константа скорости реакции принималась минимальной из исходных узких фракций для каждой новой расширенной фракции псевдокомпонента. Например, при объединении четырех псевдокомпонентов исходных узких фракций с эффективными константами скорости реакции 5, 6, 7 и 8 при расчете расширенной узкой фракции использовалась константа, равная 5, что несколько увеличивало продолжительность реакции и, опосредовано, необходимую загрузку катализатора при гарантированной степени очистки этой фракции.

В таблице 5 приведены параметры реакторов Р-1 и P-2 для реконструированного блока (таблица 4) при характеристике сырья различным числом узких фракций псевдокомпонентов. Моделирование процесса гидроочистки дизельного топлива показало, что при переходе представления сырья от 16 к 4 узким фракциям расчетная загрузка катализатора увеличивается на 30 \%, оставаясь при этом все равно почти в два раза меньше, чем при детальном расчете единичного реактора по 16 узким фракциям.

Полученные результаты хорошо согласуются с данными работы [25], рекомендующей при моделировании процесса гидроочистки использовать 5-6 псевдокомпонентов. Поскольку при уменьшении числа псевдокомпонентов и узких фракций необходимо обеспечивать снижение погрешности расчетов, для определения границы деления сырья на легкую и тяжелую фракции можно рекомендовать следующие варианты деления сырья на фракции по ИТК по мере роста температур кипения фракций:

- при делении сырья на 4 фракции: 60 \%, $10 \%, 10 \%, 10$ \%; 
- при делении сырья на 5 фракций: $50 \%, 10 \%, 10 \%, 10 \%, 10 \%$;

- при делении сырья на 6 фракций:60 \%,8 \%, 8 \%, $8 \%, 8 \%, 8 \%$,

что должно приблизить результаты расчетов, в которых принято умеренное число фракций, к варианту с 16 равными узкими фракциями псевдокомпонентов.

Таблица 5. Влияние числа узких фракций на результаты моделирования двухреакторного блока установки гидроочистки с раздельным гидрированием сырья

\begin{tabular}{|c|c|c|c|c|c|c|c|c|}
\hline \multirow[b]{2}{*}{ 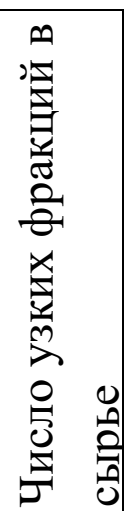 } & \multicolumn{3}{|c|}{ Реактор Р-1 } & \multicolumn{3}{|c|}{ Реактор Р-2 } & \multirow[b]{2}{*}{ 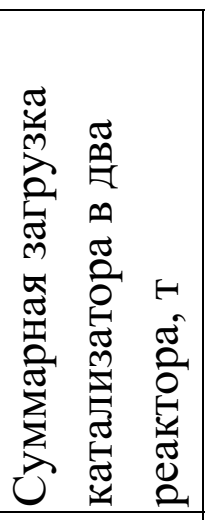 } & \multirow{2}{*}{ 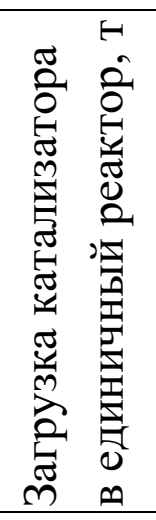 } \\
\hline & 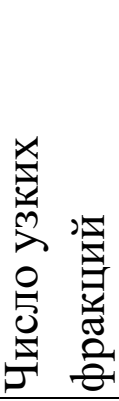 & 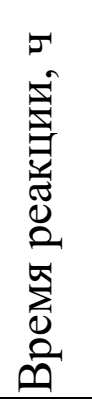 & 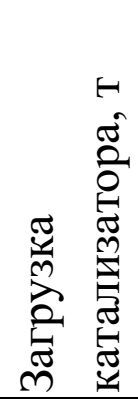 & 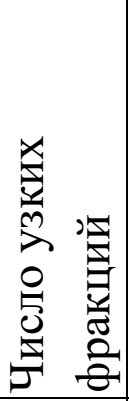 & 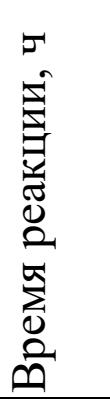 & 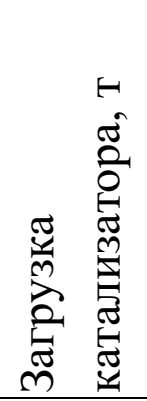 & & \\
\hline 16 & 13 & 1.1 & 44.7 & 3 & 6.2 & 58.3 & 103.0 & 227.0 \\
\hline 8 & 7 & 1.7 & 73.9 & 1 & 7.2 & 45.3 & 119.2 & 258.6 \\
\hline 4 & 3 & 1.1 & 42.3 & 1 & 7.1 & 89.2 & 131.5 & 287.6 \\
\hline
\end{tabular}

\section{Выводы}

1. Предлагается при математическом моделировании процесса гидроочистки характеристику сырья по сераорганическим веществам представлять в форме псевдокомпонента, содержащегося в узких фракциях сырья.

2. Разработана математическая модель гидроочистки дизельного топлива с использованием псевдокомпонентов.

3. Выполнена верификация математической модели гидроочистки дизельного топлива путем сравнения результатов моделирования и параметров работы промышленной установки. 
4. Дано теоретическое обоснование эффективности реализации гидроочистки дизельного топлива с индивидуальным гидрированием предварительно разделенного исходного сырья, обеспечивающей при прочих равных условиях глубокую очистку дизельного топлива при меньшей загрузке катализатора по сравнению с распространенными схемами гидроочистки всего сырья.

\section{Список используемых источников}

1. Ахметов С.А. Технология глубокой переработки нефти и газа. Уфа: Гилем, 2002. 672 с.

2. Каминский Э.Ф., Хавкин В.А. Глубокая переработка нефти: технологический и экологический аспекты. М.: Техника, 2001. 384 с.

3. Крылов В.А. Совершенствование процессов гидроочистки дизельных топлив и каталитического риформинга бензиновых фракций для переработки вторичных дистиллятов: дис. ... канд. техн. наук. Пермь: Всерос. НИИ по перераб. нефти, 2007. 160 с.

4. Танатаров М.А., Ахметшина М.Н., Фасхутдинов Р.А., Волошин Н.Д., Золотарев П.А. Технологические расчеты установок переработки нефти. М.: Химия, 1987. 350 с.

5. Кривцова Н.И., Иванчина Э.Д., Занин И.В., Ландль Ю.И., Татаурщиков А.А. Кинетические закономерности превращения серосодержащих соединений в процессе гидроочистки дизельной фракции нефти // Известия Томского политехнического университета. 2013. Т. 322. № 3. C. 83-86.

6. Song C. An Overview of New Approaches to Deep Desulfurization for Ultra-Clean Gasoline, Diesel Fuel and Jet Fuel // Catalysis Today. 2003. Vol. 86. Issue 1-4. P. 211-263. DOI: 10.1016/S0920-5861(03)00412-7. 
7. Гаврилов Н.В., Дуров О.В., Сорокин Ю.Б., Сыркин А.М. Определение причин повышенного содержания серы в продукте гидроочистки сырья риформинга // Башкирский химический журнал. 2008. Т. 15. № 2. C. 110-113.

8. Иванова Л.С., Илалдинов И.З. Проектирование установки гидроочистки дизельного топлива // Вестник Казанского технологического университета. 2013. Т. 16. № 7. С. 229-230.

9. Канашевич Д.А., Федущак Т.А., Петренко Т.В. Гидрообессеривание дизельной фракции в присутствии катализаторов, полученных с помощью механохимической активации // Известия Томского политехнического университета. 2010. Т. 317. № 3. С. 58-61.

10. Солманов П.С., Максимов Н.М., Еремина Ю.В., Жилкина Е.О., Дряглин Ю.Ю., Томина Н.И. Гидроочистка смесей дизельных фракций с бензином и легким газойлем коксования // Нефтехимия. 2013. Т. 53. № 3. C. 199-202. DOI: 10.7868/S0028242113030118.

11. Нагиев Р.С., Чернов Е.Б. Разработка современных отечественных носителей для катализаторов гидроочистки на основе $\mathrm{y}-\mathrm{Al} 2 \mathrm{O} 3$ // Башкирский химический журнал. 2015. Т. 22. № 2. С. 38-40.

12. Руденко А.В. Повышение эффективности процесса гидроочистки дизельного топлива // Актуальные проблемы гуманитарных и естественных наук. 2014. № 5-1. С. 25-27.

13. Фомиченко И.В., Ускач Я.Л. Совершенствование процесса гидроочистки дизельного топлива // Международный журнал прикладных и фундаментальных исследований. 2010. № 8. С. 145-146.

14. Жилина В.А., Самойлов Н.А. Направления модернизации установок гидроочистки дизельного топлива // Электронный научный журнал «Нефтегазовое дело». 2017. № 2. С. 90-109. URL: http://ogbus.ru/files/ogbus/issues/2_2017/ogbus_2_2017_p90-109_ZhilinaVA_ ru.pdf (дата обращения: 18.12.2020). DOI: 10.17122/ogbus-2017-2-90-109. 
15. Афанасьева Ю.И., Кривцова Н.И., Иванчина Э.Д., Занин И.К., Татаурщиков А.А. Разработка кинетической модели процесса гидроочистки дизельного топлива // Известия Томского политехнического университета. 2012. T. 321. № 3. С. 121-125.

16. Кривцова Н.И., Кривцов Е.Б., Иванчина Э.Д., Головко А.К. Кинетические закономерности гидрообессеривания дизельной фракции // Фундаментальные исследования. 2013. № 8-3. С. 640-644.

17. Xun Tang, Shuyuan Li, Changtao Yue, Jilai He, Jili Hou Lumping Kinetics of Hydrodesulfurization and Hydrodenitrogenation of the Middle Distillate from Chinese Shale Oil // Oil Shale. 2013. Vol. 30. No. 4. P. 517-535.

18. Самойлов Н.А. Анализ схем углубления гидроочистки дизельного топлива // Технология-2019: Матеріали XXII Міжнародноіі науковотехничноі конференціi. Северодонецк: СГУ, 2019. Ч. 1. С. $22-24$.

19. Пат. 2372380 РФ, МПК С 10 G 45/06. Способ селективной очистки бензиновых фракций каталитического крекинга (варианты) / В.К. Смирнов, К.Н. Ирисова, Е.Л. Талисман. 2008130976/04, Заявлено 29.07.2008; Опубл. 10.11.2009. Бюл. 31.

20. Лебедев Б.Л., Логинов С.А., Коган О.Л., Лобзин Е.В., Капустин В.М., Луговской А.И., Рудяк К.Б. Исследование состава и реакционноспособности сернистых соединений в процессе гидрообессеривания на промышленной установке // Нефтепереработка и нефтехимия. Научно-технические достижения и передовой опыт. 2001. № 11. С. 62-67.

21. Логинов С.А., Лебедев Б.Л., Капустин В.М., Луговской А.И., Курганов В.М., Рудяк К.Б. Разработка новой технологии процесса гидрообессеривания дизельных топлив // Нефтепереработка и нефтехимия. Научно-технические достижения и передовой опыт. 2001. № 11. С. 67-74.

22. Логинов С.А. Совершенствование технологии промышленного производства высококачественных дизельных топлив: дис. ... канд. техн. наук. Рязань: Рязанский нефтеперерабатывающий завод, 2002. 200 с. 
23. Пат. 2691965 РФ, МПК С 10 G 65/14. Способ гидроочистки дизельного топлива / И.А. Мнушкин, Н.А. Самойлов, В.А. Жилина. 2019102093, Заявлено 25.01.2019; Опубл. 19.06.2019. Бюл. 17.

24. Логинов С.А., Капустин В.М., Луговской А.И., Рудяк К.Б., Лебедев Б.Л. Промышленное производство высококачественных дизельных топлив с содержанием серы 0.035 и 0.05 \% // Нефтепереработка и нефтехимия. Научно-технические достижения и передовой опыт. 2001. № 11. С. 57-61.

25. Bannatham P., Teeraboonchaikul S., Patirupanon T., Arkardvipart W., Limtrakul S., Vatanatham T., Ramachandran P.A. Kinetic Evaluation for Hydrodesulfurization via Lumped Model in a Trickle-Bed Reactor // Industrial and Engineering Chemistry Research. 2016. Vol. 55. No. 17. P. 4878-4886. DOI: 10.1021/acs.iecr.6b00382.

\section{References}

1. Akhmetov S.A. Tekhnologiya glubokoi pererabotki nefti i gaza [Deep Processing Technology of Oil and Gas]. Ufa, Gilem Publ., 2002. 672 p. [in Russian].

2. Kaminskii E.F., Khavkin V.A. Glubokaya pererabotka nefti: tekhnologicheskii i ekologicheskii aspekty [Deep Refining of Oil: Technological and Environmental Aspects]. Moscow, Tekhnika Publ., 2001. 384 p. [in Russian].

3. Krylov V.A. Sovershenstvovanie protsessov gidroochistki dizel'nykh topliv $i$ kataliticheskogo riforminga benzinovykh fraktsii dlya pererabotki vtorichnykh distillyatov: dis. kand. tekhn. nauk [Improving the Processes of Diesel Fuel Hydrotreating and Catalytic Reforming of Gasoline Fractions for Processing Secondary Distillates: Cand. Engin. Sci. Diss.]. Perm, Vseros. NII po pererab. nefti Publ., 2007. 160 p. [in Russian]. 
4. Tanatarov M.A., Akhmetshina M.N., Faskhutdinov R.A., Voloshin N.D., Zolotarev P.A. Tekhnologicheskie raschety ustanovok pererabotki nefti [Technological Calculations of Oil Refining Units]. Moscow, Khimiya Publ., 1987. 350 p. [in Russian].

5. Krivtsova N.I., Ivanchina E.D., Zanin I.V., Landl' Yu.I., Tataurshchikov A.A. Kineticheskie zakonomernosti prevrashcheniya serosoderzhashchikh soedinenii $\mathrm{v}$ protsesse gidroochistki dizel'noi fraktsii nefti [Kinetic Regularities of the Conversion of Sulfur-Containing Compounds in the Process of Hydrotreating the Diesel Fraction of Oil]. Izvestiya Tomskogo politekhnicheskogo universiteta - Bulletin of the Tomsk Polytechnic University, 2013, Vol. 322, No. 3, pp. 83-86. [in Russian].

6. Song C. An Overview of New Approaches to Deep Desulfurization for Ultra-Clean Gasoline, Diesel Fuel and Jet Fuel. Catalysis Today, 2003, Vol. 86, Issue 1-4, pp. 211-263. DOI: 10.1016/S0920-5861(03)00412-7.

7. Gavrilov N.V., Durov O.V., Sorokin Yu.B., Syrkin A.M. Opredelenie prichin povyshennogo soderzhaniya sery $\mathrm{v}$ produkte gidroochistki syr'ya riforminga [Definition of the Reasons of the Raised Contents of Sulfur at Hydroclearing Reforming Feed]. Bashkirskii khimicheskii zhurnal - Bashkir Chemical Journal, 2008, Vol. 15, No. 2, pp. 110-113. [in Russian].

8. Ivanova L.S., Ilaldinov I.Z. Proektirovanie ustanovki gidroochistki dizel'nogo topliva [Design of a Diesel Hydrotreater]. Vestnik Kazanskogo tekhnologicheskogo universiteta - Herald of Kazan Technological University, 2013, Vol. 16, No. 7, pp. 229-230. [in Russian].

9. Kanashevich D.A., Fedushchak T.A., Petrenko T.V. Gidroobesserivanie dizel'noi fraktsii $\mathrm{v}$ prisutstvii katalizatorov, poluchennykh $\mathrm{s}$ pomoshch'yu mekhanokhimicheskoi aktivatsii [Hydrodesulfurization of the Diesel Fraction in the Presence of Catalysts Obtained by Mechanochemical Activation]. Izvestiya Tomskogo politekhnicheskogo universiteta - Bulletin of the Tomsk Polytechnic University, 2010, Vol. 317, No. 3, pp. 58-61. [in Russian]. 
10. Solmanov P.S., Maksimov N.M., Eremina Yu.V., Zhilkina E.O., Dryaglin Yu.Yu., Tomina N.I. Gidroochistka smesei dizel'nykh fraktsii s benzinom i legkim gazoilem koksovaniya [Hydrotreating of Mixtures of Diesel Fractions with Gasoline and Light Coker Gas Oil]. Neftekhimiya - Neftekhimiya, 2013, Vol. 53, No. 3, pp. 199-202. DOI: 10.7868/S0028242113030118. [in Russian].

11. Nagiev R.S., Chernov E.B. Razrabotka sovremennykh otechestvennykh nositelei dlya katalizatorov gidroochistki na osnove y-Al2O3 [Development of Modern Domestic Carriers for Hydrotreating Catalysts on the Base of $\gamma$-AL2O3]. Bashkirskii khimicheskii zhurnal - Bashkir Chemical Journal, 2015, Vol. 22, No. 2, pp. 38-40. [in Russian].

12. Rudenko A.V. Povyshenie effektivnosti protsessa gidroochistki dizel'nogo topliva [Improving the Efficiency of the Diesel Hydrotreating Process]. Aktual'nye problemy gumanitarnykh i estestvennykh nauk - Actual Problems of the Humanities and Natural Sciences, 2014, No. 5-1, pp. 25-27. [in Russian].

13. Fomichenko I.V., Uskach Ya.L. Sovershenstvovanie protsessa gidroochistki dizel'nogo topliva [Improving the Process of Diesel Fuel Hydrotreating]. Mezhdunarodnyi zhurnal prikladnykh $i$ fundamental'nykh issledovanii - International Journal of Applied and Fundamental Research, 2010, No. 8, pp. 145-146. [in Russian].

14. Zhilina V.A., Samoilov N.A. Napravleniya modernizatsii ustanovok gidroochistki dizel'nogo topliva [Directions of Modernization of Diesel Fuel Hydrotreating Plants]. Elektronnyi nauchnyi zhurnal «Neftegazovoe delo»Electronic Scientific Journal «Oil and Gas Business», 2017, No. 2, pp. 90-109. URL: ～http://ogbus.ru/files/ogbus/issues/2_2017/ogbus_2_2017_p90-109_ ZhilinaVA_ru.pdf (accessed 18.12.2020). DOI: 10.17122/ogbus-2017-2-90-109. [in Russian]. 
15. Afanaseva Yu.I., Krivtsova N.I., Ivanchina E.D., Zanin I.K., Tataurshchikov A.A. Razrabotka kineticheskoi modeli protsessa gidroochistki dizel'nogo topliva [Development of a Kinetic Model of the Diesel Fuel Hydrotreating Process]. Izvestiya Tomskogo politekhnicheskogo universiteta Bulletin of the Tomsk Polytechnic University, 2012, Vol. 321, No. 3, pp. 121-125. [in Russian].

16. Krivtsova N.I., Krivtsov E.B., Ivanchina E.D., Golovko A.K. Kineticheskie zakonomernosti gidroobesserivaniya dizel'noi fraktsii [Kinetic Regularities of Hydro-Desulfurization of Diesel Fuel]. Fundamental'nye issledovaniya - Fundamental Research, 2013, No. 8-3, pp. 640-644. [in Russian].

17. Xun Tang, Shuyuan Li, Changtao Yue, Jilai He, Jili Hou Lumping Kinetics of Hydrodesulfurization and Hydrodenitrogenation of the Middle Distillate from Chinese Shale Oil. Oil Shale, 2013, Vol. 30, No. 4, pp. 517-535.

18. Samoilov N.A. Analiz skhem uglubleniya gidroochistki dizel'nogo topliva [Analysis of Schemes for Deepening Hydrotreating of Diesel Fuel]. Materiali XXII Mizhnarodnoii naukovo-tekhnichnoi konferentsii «Tekhnologiya-2019» [Proceedings of the XXII International Scientific and Technical Conference «Technology-2019»]. Severodonetsk, SGU Publ., 2019, Part 1, pp. 22-24. [in Russian].

19. Smirnov V.K., Irisova K.N., Talisman E.L. Sposob selektivnoi ochistki benzinovykh fraktsii kataliticheskogo krekinga (varianty) [Method for Selective Purification of Gasoline Fractions of Catalytic Cracking (Versions)]. Patent RF, No. 2372380, 2009. [in Russian].

20. Lebedev B.L., Loginov S.A., Kogan O.L., Lobzin E.V., Kapustin V.M., Lugovskoi A.I., Rudyak K.B. Issledovanie sostava i reaktsionnosposobnosti sernistykh soedinenii $\mathrm{v}$ protsesse gidroobesserivaniya na promyshlennoi ustanovke [Study of the Composition and Reactivity of Sulfur Compounds in the Process of Hydrodesulfurization at an Industrial Plant]. Neftepererabotka $i$ neftekhimiya. Nauchno-tekhnicheskie dostizheniya i peredovoi opyt - Oil Processing and Petrochemistry, 2001, No. 11, pp. 62-67. [in Russian]. 
21. Loginov S.A., Lebedev B.L., Kapustin V.M., Lugovskoi A.I., Kurganov V.M., Rudyak K.B. Razrabotka novoi tekhnologii protsessa gidroobesserivaniya dizel'nykh topliv [Development of a New Technology for the Process of Hydrodesulfurization of Diesel Fuels]. Neftepererabotka $i$ neftekhimiya. Nauchno-tekhnicheskie dostizheniya i peredovoi opyt - Oil Processing and Petrochemistry, 2001, No. 11, pp. 67-74. [in Russian].

22. Loginov S.A. Sovershenstvovanie tekhnologii promyshlennogo proizvodstva vysokokachestvennykh dizel'nykh topliv: dis. kand. tekhn. nauk [Improvement of Technology for Industrial Production of High Quality Diesel Fuels: Cand. Engin. Sci. Diss.]. Ryazan, Ryazanskii neftepererabatyvayushchii zavod Publ., 2002. 200 p. [in Russian].

23. Mnushkin I.A., Samoilov N.A., Zhilina V.A. Sposob gidroochistki dizel'nogo topliva [Diesel Fuel Hydrotreating Method]. Patent RF, No. 2691965, 2019. [in Russian].

24. Loginov S.A., Kapustin V.M., Lugovskoi A.I., Rudyak K.B., Lebedev B.L. Promyshlennoe proizvodstvo vysokokachestvennykh dizel'nykh topliv s soderzhaniem sery 0.035 i $0.05 \%$ [Industrial Production of High Quality Diesel Fuels with a Sulfur Content of 0.035 and 0.05\%]. Neftepererabotka $i$ neftekhimiya. Nauchno-tekhnicheskie dostizheniya i peredovoi opyt - Oil Processing and Petrochemistry, 2001, No. 11, pp. 57-61. [in Russian].

25. Bannatham P., Teeraboonchaikul S., Patirupanon T., Arkardvipart W., Limtrakul S., Vatanatham T., Ramachandran P.A. Kinetic Evaluation for Hydrodesulfurization via Lumped Model in a Trickle-Bed Reactor. Industrial and Engineering Chemistry Research, 2016, Vol. 55, No. 17, pp. 4878-4886. DOI: 10.1021/acs.iecr.6b00382. 


\section{Сведения об авторах}

\section{About the authors}

Самойлов Наум Александрович, д-р техн. наук, профессор кафедры «Нефтехимия и химическая технология», УГНТУ, г. Уфа, Российская Федерация

Naum A. Samoilov, Doctor of Engineering Sciences, Professor of Petrochemistry and Chemical Technology Department, USPTU, Ufa, Russian Federation

e-mail: naum.samoilow@yandex.ru

Жилина Валерия Анатольевна, аспирант кафедры «Нефтехимия и химическая технология», УГНТУ, г. Уфа, Российская Федерация

Valeria A. Zhilina, Post-graduate Student of Petrochemistry and Chemical Technology Department, USPTU, Ufa, Russian Federation

e-mail: ofelia90@mail.ru 